ARTIGO

\title{
Associação entre bullying escolar e o país de origem: um estudo transcultural
}

\author{
Marcela Almeida Zequinão' (D) \\ Pâmella de Medeiros' (D \\ Fábio Augusto Lise" (D) \\ Maria Teresa Ceron Trevisol" (D) \\ Maria Beatriz Ferreira Leite Oliveira Pereira"I D
}

\section{RESUMO}

O objetivo do estudo foi analisar o bullying em Portugal e no Brasil verificando possíveis associações com o país de origem. Participaram 789 crianças e adolescentes. Apesar de haver diversos questionários acerca da temática, utilizou-se o Questionário de Olweus em virtude de sua relevância e vasta utilização em todo o mundo, o que facilita a comparação dos resultados. Verificou-se que os brasileiros apresentaram associações envolvendo menor escolaridade da mãe, maior índice de reprovação escolar, menor incidência da prática de atividades físicas, menos chances de denunciar o agressor e menos apoio dos colegas no momento da agressão. Os brasileiros se autodeclararam mais vítimas e os portugueses mais vítimas-agressoras. Em ambos os países, o local em que mais aconteceram agressões foi no recreio. Em geral, os brasileiros apresentaram mais indícios de vulnerabilidade para o envolvimento em situações de bullying, ressaltando-se o isolamento social como característica de risco das vítimas-agressoras.

PALAVRAS-CHAVE

bullying; vitimização; agressão; vítimas-agressoras; transcultural.

'Universidade do Estado de Santa Catarina, Florianópolis, SC, Brasil.

"Universidade do Oeste de Santa Catarina, Joaçaba, SC, Brasil.

"'Universidade do Minho, Braga, Portugal. 


\title{
ASSOCIATION BETWEEN SCHOOL BULLYING AND COUNTRY OF ORIGIN: A TRANSCULTURAL STUDY
}

\begin{abstract}
The aim of the study was to analyze bullying in Portugal and Brazil and to verify possible associations with the country of origin. 789 children and adolescents participated. The Olweus Questionnaire was used to analyze bullying. Although there are several questionnaires in this theme, it was chosen due to its relevance and wide use throughout the world, which facilitates the comparison of the results. It was verified that Brazilians presented associations with less education of mother, greater disapproval, less physical activity practice, less chances of denouncing the aggressor and less support of the peers at the time of the aggression. Brazilians declared themselves more victims and the Portuguese more victims-aggressors. In both countries the place where the most aggressions occurred was in the playground. In general, Brazilians presented greater evidence of vulnerability to bullying involvement, and social isolation was highlighted as a risk characteristic of victim-aggressors.
\end{abstract}

KEYWORDS

bullying; victimization; aggression; bully-victim; transcultural.

\section{ASOCIACIÓN ENTRE BULLYING ESCOLAR Y EL PAÍS DE ORIGEN: UN ESTUDIO TRANSCULTURAL}

\section{RESUMEN}

El objetivo del estudio fue analizar la intimidación en Portugal y Brasil y verificar posibles asociaciones con el país de origen. Participaron 789 niños y adolescentes. Se utilizó el Cuestionario de Olweus para el análisis del bullying. A pesar de haber varios cuestionarios en esta temática, éste fue escogido en función de su relevancia y amplia utilización en todo el mundo, lo que facilita la comparación de los resultados. Los brasileños presentaron asociaciones con menor escolaridad de la madre, mayor reprobación, menor práctica de actividades físicas, menos posibilidades de denunciar al agresor y menos apoyo de los colegas en el momento de la agresión. Los brasileños se declararon más víctimas y los portugués más víctimas-agresoras. En ambos países el lugar en que ocurrieron más agresiones fue en el recreo. Em general, los brasileños presentaron mayores indicios de vulnerabilidad para el bullying y se resalta el aislamiento social como característica de riesgo de las víctimas-agresoras.

PALABRAS CLAVE

bullying; victimización; agresión; víctimas-agresores; transcultural. 


\section{INTRODUÇÃO}

O bullying escolar vem sendo uma temática bastante discutida na literatura mundial nas últimas décadas. Sabe-se que esse fenômeno se tornou um problema de saúde pública, considerando sua elevada prevalência e os prejuízos que causa ao desenvolvimento saudável e ao bem-estar psicossocial de crianças e adolescentes (De Oliveira et al., 2015). Atualmente, pode-se dizer que há um consenso sobre a definição de bullying, que é caracterizado como qualquer comportamento agressivo constituído de três elementos centrais: a intenção de prejudicar a vítima; a natureza repetitiva das agressões; e o desequilíbrio de poder entre o agressor e a vítima (Centers for Disease Control and Prevention, 2014; Gaffney, Ttofi e Farrington, 2018). É também um fenômeno social e de grupo, no qual os comportamentos dos estudantes envolvidos, bem como dos demais membros da comunidade escolar, exercem efeito sobre a sua gênese, manutenção e/ou interrupção. Sendo assim, o bullying pode envolver crianças e adolescentes de diferentes maneiras, fazendo com que estas assumam papéis diferenciados em relação à postura adotada perante o acontecimento.

No entanto, apesar de a ocorrência do bullying escolar ser considerada bastante comum, ainda permanece na literatura certo grau de discordância em relação ao percentual de envolvimento nesses eventos, principalmente em virtude dos métodos de avaliação utilizados nas pesquisas (Gaffney, Ttofi e Farrington, 2018). Em um estudo realizado em 40 países para verificar a prevalência de bullying, identificou-se que, em média, 26\% dos adolescentes estão envolvidos nessa situação (Craig et al., 2009), enquanto uma recente metanálise de 80 estudos internacionais descobriu a prevalência de 34,5 e 36\% para agressores e vítimas de bullying, respectivamente (Modecki et al., 2014).

Entre esses papéis de participação, podem-se citar o agressor e a vítima (Tsang, Hui e Law, 2011), sendo que muitas vezes esses papéis acabam entrelaçados, situação na qual um mesmo indivíduo pode assumir duas ou mais posturas, como no caso das vítimas-agressoras (Kochel et al., 2015). Além dessas atuações, tem-se também o espectador, que pode ser tanto defensor quanto reforçador desse tipo de violência (Zych et al., 2017). Sabe-se que, independentemente do papel assumido, a participação nesse acontecimento pode trazer diversas consequências negativas aos envolvidos, resultando em problemas graves até mesmo na vida adulta (Obrdalj et al., 2013).

Considerando esse aspecto, muitos pesquisadores da área desenvolveram programas de prevenção, de intervenção e combate ao bullying nas escolas. Craig et al. (2009) apontam que grande parte desses estudos não vinha encontrando sucesso nos resultados verificados, fato decorrente da aplicação das intervenções propostas na literatura. Contudo, uma recente metanálise, que incluiu 100 avaliações de intervenção contra o bullying nas escolas, com diversos modelos de programas antibullying baseados na escola, verificou que de modo geral os programas reduzem efetivamente o bullying escolar em relação à agressão (aproximadamente 19-20\%) e à vitimização (15-16\%) (Gaffney, Ttofi e Farrington, 2018). 
A eficácia dos programas de intervenção também pode ser explorada de acordo com as características da avaliação, como a localização de implementação. Para exemplificar tal situação, podem-se citar os achados com dois dos principais programas de intervenção conhecidos mundialmente: o KiVa Antibullying Program (Salmivalli e Poskiparta,2011) e o Olweus Bullying Prevention Program (OBPP) (Olweus, 1991). Embora ambos os programas tenham sido aplicados em diferentes países e obtidos resultados positivos em relação ao combate ao bullying, percebe-se que os efeitos foram maiores nos estudos realizados com o KiVa na Finlândia e com o OBPP na Noruega (seus países de origem) do que quando foram realizados em outros países (Gaffney, Ttofi e Farrington, 2018). Assim, Molcho et al. (2009) sugerem que pesquisas futuras devem explorar como a eficácia dos programas antibullying pode variar em todo o mundo, sendo necessário identificar quais componentes específicos dos programas de intervenção são mais eficientes na redução do bullying em cada contexto.

Acredita-se que programas de intervenção generalistas e com uma visão descontextualizada da realidade da escola não conseguem solucionar as dificuldades encontradas no ambiente escolar. Ou seja, para se compreender esse tipo de violência é preciso considerar que o ecossistema escolar está envolvido em sua origem e manutenção. Qualquer tipo de interferência no bullying deve levar em consideração diversas dimensões sociais, além de uma visão ecológica sobre sua propagação (Bronfenbrenner, 2011; Freire e Aires, 2012).

Bronfenbrenner (2011) traz importantes contribuições acerca da interferência do meio no desenvolvimento dos indivíduos considerando sua teoria bioecológica do desenvolvimento humano. Segundo esse autor, quatro aspectos se inter-relacionam na constituição do desenvolvimento do sujeito, sendo eles: processo, pessoa, contexto e tempo, conhecidos como modelo PPCT. O processo refere-se à relação entre as características da pessoa e do contexto, sendo importantes aqui os processos proximais, aqueles caracterizados por interações recíprocas, estáveis e duradouras entre a pessoa, os objetos e os símbolos do seu ambiente externo imediato. A pessoa é entendida como um sujeito biopsicossocial, constituída de componentes individuais, biológicos, hereditários, cognitivos, emocionais e comportamentais.

Sendo um fenômeno de extrema complexidade, para ser mais bem compreendido em suas diversas facetas, o bullying escolar precisa ser investigado de maneira profunda, principalmente em diferentes comunidades (Zequinão et al., 2016). Acredita-se que com a recolha de dados envolvendo diversidades seja possível a elaboração de programas de intervenção mais eficazes, que visem analisar o bullying e seus envolvidos em diferentes contextos e culturas. Entretanto, faz-se necessário desenvolver estudos de modelos transculturais e permitir o estabelecimento de um quadro mais completo das determinantes de bullying entre crianças e adolescentes (Harel-Fisch et al., 2011).

Considerando esses aspectos, este artigo apresenta como base empírica uma investigação que buscou analisar a compreensão de como se comportam os envolvidos no fenômeno bullying em diferentes realidades socioculturais, bem como estabelecer a ecologia desse fenômeno, mapear as relações sociais entre os atores envolvidos, os comportamentos, afetos e silêncios que retroalimentam e contribuem para a manutenção do ciclo de violência. Constitui objetivo deste artigo analisar o bullying escolar em Portugal e no Brasil e verificar possíveis associações com o país de origem. 


\section{MÉTODOS}

\section{DESIGN}

A pesquisa realizada, base empírica deste artigo, caracteriza-se como um estudo transcultural, de corte transversal, de cunho exploratório e amostragem intencional, realizado na cidade metropolitana de Braga, Região do Minho, Norte de Portugal, e na cidade metropolitana de Florianópolis, estado de Santa Catarina, Região Sul do Brasil, entre novembro de 2014 e maio de 2015. Esta pesquisa faz parte de um macroprojeto aprovado no Comitê de Ética de Ciências Sociais e Humanas da Universidade do Minho, processo n. 010/2014 (Portugal), e no Comitê de Ética em Pesquisas com Seres Humanos da Universidade do Estado de Santa Catarina (UDESC), processo n. 5.439/2011, expediente n. 75/2011 (Brasil).

Apesar de Portugal e Brasil serem países lusófonos, a escolha por tais espaços deu-se em virtude de se considerar que ambos possuem características muito específicas em relação a questões sociais, econômicas, políticas e culturais que podem refletir também de maneira diferenciada dentro do contexto escolar. Dessa forma, o objetivo geral da pesquisa foi analisar o bullying escolar em Portugal e no Brasil e verificar possíveis associações com o país de origem (Portugal e Brasil). Para isso, o artigo foi estruturado com base nos seguintes objetivos específicos:

- comparar as características gerais dos participantes de ambos os países (com base em questões como nível de escolaridade da mãe; reprovação escolar; prática de atividade física; perfil de participação no bullying);

- comparar as características dos processos referentes ao bullying em ambos os países (com base em informações das vítimas sobre contar a alguém a respeito da agressão sofrida e do apoio recebido; principais lugares onde ocorrem as vitimizações; frequência que agrediu alguém na escola e os motivos; frequência sobre ajudar a agredir um colega; associação entre papéis de participação no bullying e experiência de ficar isolado na escola).

\section{PARTICIPANTES E PROCEDIMENTOS}

A seleção das escolas em ambos os países foi intencional, de acordo com aquelas que demonstraram interesse e disponibilidade em participar da pesquisa. Com isso, integraram o estudo quatro escolas públicas municipais e uma escola privada em Braga, e uma escola pública municipal e duas escolas privadas em Florianópolis. Após a seleção, foram convidadas a participar da pesquisa todas as crianças e adolescentes do $3^{\circ}$ ao $6^{\circ}$ ano do ensino fundamental, de ambos os sexos, matriculadas no período vigente do estudo.

Com base no número total de crianças e adolescentes que estavam cursando entre o $3^{\circ}$ e $6^{\circ}$ ano nas duas cidades e suas respectivas proporcionalidades entre escolas públicas e privadas, um tamanho de amostra foi calculado assumindo um alfa de 0,05 e poder de $50 \%$. De acordo com o cálculo amostral, chegou-se ao número de 767 
participantes, sendo 374 em Portugal (299 de escola pública e 75 de escola privada) e 393 no Brasil (266 de escola pública e 126 de escola privada). Tendo em vista que todas as crianças e adolescentes do $3^{\circ}$ ao $6^{\circ}$ ano foram convidadas a participar, o número de participantes total foi de 789, sendo 392 em Portugal (308 de escola pública e 84 de escola privada) e 397 no Brasil (268 de escola pública e 129 de escola privada).

Previamente à coleta dos dados, os pais e as crianças e adolescentes receberam informações detalhadas sobre a pesquisa. Os participantes foram devidamente esclarecidos sobre o estudo e explicitaram sua anuência em participar por meio do Termo de Assentimento (TA), que foi elaborado em linguagem clara e acessível para os menores de idade. Da mesma forma foi enviado aos pais e/ou responsáveis o Termo de Consentimento Livre e Esclarecido (TCLE), documento no qual é explicitado o consentimento do responsável legal da criança.

Após a entrega dos termos de consentimento e assentimento assinados, todos os participantes responderam ao questionário de autorrelato sobre sua participação em situações de bullying, o qual foi aplicado e supervisionado por dois pesquisadores treinados para esclarecer qualquer dúvida dos participantes.

O único critério de exclusão adotado no recrutamento dos participantes foi apresentar alguma deficiência intelectual que impedisse a compreensão dos instrumentos, com base na indicação do Atendimento Escolar Especial (AEE) das escolas.

\section{VARIÁVEIS}

Para descrever as situações de bullying e os possiveis papéis de participação nesse fenômeno foi utilizado o Questionário de Olweus (1996). Por meio de duas questões foi possível estabelecer o perfil dos participantes. A primeira questão perguntava quantas vezes, nos últimos três meses de aula, o participante tinha sido vítima de bullying escolar, enquanto a segunda perguntava quantas vezes o participante tinha sido agressor. Com as informações obtidas por meio daqueles que responderam que foram vítimas e agressores três ou mais vezes, criou-se uma variável "vítima-agressora". Assim, os participantes foram classificados em quatro categorias de participação no bullying:

1. "não participa";

2. "vítima";

3. "agressor"; e

4. "vítima-agressora".

O Questionário de Olweus apresentou nível de consistência interna moderado, com alfa de Cronbach igual a 0,614.

\section{ANÁLISE ESTATÍSTICA}

Os dados foram analisados inicialmente pela estatística descritiva (média, desvio padrão e distribuição de frequência). A normalidade dos dados foi verificada por meio do teste Kolmogorov-Smirnov. O teste U de Mann-Whitney foi utilizado para verificar as diferenças entre os grupos, e o teste qui-quadrado verificou as associações entre as variáveis categóricas. Em todas as análises adotou-se o nível de significância de 5\%, utilizando o programa estatístico SPSS, versão 20.0. 


\section{RESULTADOS}

\section{ANÁLISE COMPARATIVA DA CARACTERIZAÇÃO DOS PARTICIPANTES}

Como se pode verificar na Tabela 1 , na caracterização dos participantes de Portugal e do Brasil, estes apresentaram homogeneidade em relação a sua idade $\left(x^{-}=9,89 ; \mathrm{DP}=1,30 ; x^{-}=9,77 ; \mathrm{DP}=1,46\right.$, respectivamente). No entanto, em todas as outras variáveis foi encontrada uma associação significativa com o país. Isso pode evidenciar que, embora existam algumas similaridades entre os dois países, os aspectos socioeconômicos, político/legislativos e culturais têm grande impacto nas relações e características psicossociais dos participantes da pesquisa.

Entre essas variáveis sociodemográficas associadas ao país encontrava-se a escolaridade das mães. Considerando as respostas aos questionários e seu lugar de origem, pode-se constatar que em Portugal, predominantemente, as mães estudaram até o ensino médio (26,3\%), enquanto no Brasil a maioria das mães possuía o ensino fundamental (33\%). Porém, ao se comparar os dados referentes ao número de mães com curso superior, percebe-se que no Brasil $(23,2 \%)$ existem mais mães com terceiro grau que em Portugal (17,1\%). Pode-se perceber maior polaridade na escolaridade das mães brasileiras, pois a maior parte delas ou possui o ensino fundamental ou nível superior (dois extremos), enquanto as mães de Portugal apresentaram níveis educacionais mais harmônicos, concentrados no ensino fundamental e médio, com menos pessoas com ensino superior. Esses dados podem ser indicativos de diferenças entre acessibilidade, investimentos governamentais e aspectos políticos do sistema educacional nas duas realidades.

Ao se analisar os dados sobre as características escolares dos participantes da pesquisa, verificou-se que na amostra brasileira houve quase quatro vezes mais reprovações $(20,4 \%)$, se comparado ao contexto português $(6,7 \%)$. Além disso, mais da metade dos estudantes do Brasil relatou que não praticava atividade física $(51,7 \%)$, quando os de Portugal $(41,4 \%)$ apresentaram um índice bem menor. Esses dados também podem ser considerados indicativos de diferenças culturais e práticas educacionais adotadas pelos dois países, pois crianças com faixa etária similar, expostas a conhecimentos do mesmo grau de complexidade, deveriam atingir o mesmo desempenho escolar. Sendo assim, pode-se considerar que no Brasil, possivelmente, exista uma deficiência nos processos integrantes da aprendizagem e/ou nos processos de sua avaliação. Além disso, a discrepância na incidência da prática de atividades físicas pode evidenciar uma diferenciação cultural e de valores entre as duas amostras que compuseram o estudo realizado.

A realização dessa comparação entre as características dos participantes da pesquisa torna-se importante para se verificar as diferenças socioeconômicas e culturais entre os dois países e de que maneira elas tem consequência nos comportamentos adotados por crianças e adolescentes em idade escolar. E, refletindo a respeito da ação escolar no enfrentamento do fenômeno bullying, cabe aos profissionais atuantes na escola planejar intervenções considerando a complexidade do problema enfrentado, sem deixar de voltar sua atenção ao contexto social dos envolvidos (Freire e Aires, 2012). 
Tabela 1 - Características gerais dos escolares de Portugal e do Brasil.

\begin{tabular}{|c|c|c|c|c|}
\hline \multirow[t]{2}{*}{ Variáveis } & $\begin{array}{c}\text { Total } \\
(\mathrm{N}=789)\end{array}$ & $\begin{array}{l}\text { Portugal } \\
(\mathrm{N}=392)\end{array}$ & $\begin{array}{c}\text { Brasil } \\
(\mathrm{N}=397)\end{array}$ & \multirow[t]{2}{*}{ p-valor* } \\
\hline & $\bar{x}(\mathrm{DP})$ & $\bar{x}(\mathrm{DP})$ & $\bar{x}(\mathrm{DP})$ & \\
\hline Idade (anos) & $9,83(1,38)$ & $9,89(1,30)$ & $9,77(1,46)$ & 0,170 \\
\hline \multirow{2}{*}{ Variáveis } & Total & Portugal & Brasil & \multirow{2}{*}{$p$-valor** } \\
\hline & $\mathrm{n}(\%)$ & $\mathrm{n}(\%)$ & $\mathrm{n}(\%)$ & \\
\hline Escolaridade da mãe & & & & $<0,001$ \\
\hline Não soube responder & $252(32,0)$ & $130(33,2)$ & $122(30,7)$ & \\
\hline Ensino fundamental & $222(28,2)$ & $91(23,3)$ & $131(33,0)$ & \\
\hline Ensino médio & $155(19,7)$ & $103(26,3)$ & $52(13,1)$ & \\
\hline Ensino superior & $159(20,2)$ & $67(17,1)$ & $92(23,2)$ & \\
\hline Repetiu de ano na escola & & & & $<0,001$ \\
\hline Não & $676(86,4)$ & $363(93,3)$ & $313(79,6)$ & \\
\hline Sim & $106(13,6)$ & $26(6,7)$ & $80(20,4)$ & \\
\hline Pratica atividade física & & & & 0,003 \\
\hline Não & $363(46,5)$ & $162(41,4)$ & $201(51,7)$ & \\
\hline Sim & $417(53,5)$ & $229(58,6)$ & $188(48,3)$ & \\
\hline Bullying & & & & 0,029 \\
\hline Não participa & $594(75,3)$ & $289(73,7)$ & $305(76,8)$ & \\
\hline Vítima & $129(16,3)$ & $60(15,3)$ & $69(17,4)$ & \\
\hline Agressor & $32(4,1)$ & $18(4,6)$ & $14(3,5)$ & \\
\hline Vítima-agressora & $34(4,3)$ & $25(6,4)$ & $9(2,3)$ & \\
\hline
\end{tabular}

$\bar{x}(\mathrm{DP})$ : média (desvio padrão); n: número de participantes. ${ }^{*} \mathrm{U}$ de Mann-Whitney. ${ }^{* *}$ Qui-quadrado. Fonte: Banco de dados da pesquisa. Elaboração dos autores.

\section{ANÁLISE COMPARATIVA DA CARACTERIZAÇÃO DOS PROCESSOS REFERENTES AO BULLYING}

Embora apenas recentemente o fenômeno bullying tenha começado a se destacar como gerador de sofrimento psíquico e violência psicológica, é importante afinar que sua ocorrência é anterior a sua visibilidade, ou seja, por muito tempo esse acontecimento não foi nominado ou caracterizado como danoso para as relações sociais e de aprendizagem na escola (Freire e Aires, 2012). Ao se analisar os dados do estudo, pode-se constatar um número elevado da incidência desse processo e violência escolar. Na Tabela 2, pode-se perceber que foi encontrada uma associação 
entre o número de vezes que os participantes sofreram agressões na escola e o país de origem $(p=0,001)$. Dessa maneira, os participantes brasileiros relataram maior frequência em vitimização leve ( $37 \%$ uma ou duas vezes), bem como em vitimização severa ( $13,4 \%$ cinco ou mais vezes). Já os portugueses relataram maior frequência em vitimização média ( $10,2 \%$ três ou quatro vezes).

O bullying é uma afirmação de poder interpessoal por meio da agressão sobre uma vítima mais fraca, apresentando efeitos imediatos e tardios nos atores envolvidos (agressores, vítimas e observadores). Os efeitos negativos são causados tanto pelas agressões como pela vitimização. Na Tabela 1, cabe destacar como os participantes da pesquisa se identificaram quanto ao seu papel nos processos que levam ao bullying. Entre os escolares brasileiros, pode-se perceber uma maior porcentagem de vítimas. $\mathrm{O}$ alvo do bullying é aquela pessoa que por repetidas vezes acaba sofrendo alguma agressão intencional; a vítima apresenta características que a tornam mais suscetível a sofrer algum tipo de violência. Muitas dessas características podem estar relacionadas a formas de tratamento familiar que ceifam a autonomia (Lopes Neto, 2005) ou que superprotegem as crianças, não as desafiando a tomar posições e assumir comportamentos de acordo com situações do cotidiano. Já entre os escolares portugueses, constatou-se maior incidência de vítimas-agressoras $(\mathrm{p}=0,029)$ (Tabela 1). Alvos/Autores são aqueles que sofrem o bullying e o replicam em outros colegas, que usam o bullying para diminuir seu sentimento de inferioridade. Dados de pesquisa têm revelado que esses indivíduos são mais propensos a problemas psíquicos (Lopes Neto, 2005).

Tabela 2 - Frequência com que sofreu vitimização na escola.

\begin{tabular}{|c|c|c|c|c|}
\hline \multirow{2}{*}{$\begin{array}{l}\text { Quantas vezes algum(a) } \\
\text { aluno(a) fez algo de mal a } \\
\text { você no último período? }\end{array}$} & Total & Portugal & Brasil & \multirow{2}{*}{ p-valor* } \\
\hline & $\mathrm{n}(\%)$ & $\mathrm{n}(\%)$ & $\mathrm{n}(\%)$ & \\
\hline Nenhuma vez & $378(47,9)$ & $206(52,6)$ & $172(43,3)$ & \multirow{4}{*}{0,001} \\
\hline Uma ou duas vezes & $245(31,1)$ & $98(25,0)$ & $147(37,0)$ & \\
\hline Três ou quatro vezes & $65(8,2)$ & $40(10,2)$ & $25(6,3)$ & \\
\hline Cinco ou mais vezes & $101(12,8)$ & $48(12,2)$ & $53(13,4)$ & \\
\hline
\end{tabular}

n: número de participantes. *Qui-quadrado. Fonte: Banco de dados da pesquisa. Elaboração dos autores.

Verificou-se também uma associação significativa entre contar a alguém sobre a vitimização e o país de origem ( $\mathrm{p}=0,012)$. Observou-se que $22,7 \%$ dos participantes brasileiros não contaram a ninguém sobre a agressão sofrida, enquanto $13,7 \%$ dos portugueses também não o fizeram. Na maioria das vezes, os alvos não falam ou reclamam sobre o bullying por medo de não serem ouvidos ou por vergonha de suas características. Geralmente o alvo não possui recursos psíquicos para reagir à agressão (Lopes Neto, 2005).

Do mesmo modo, também foi encontrada associação significativa dos países - Brasil e Portugal - em relação ao apoio recebido $(\mathrm{p}<0,001)$ em situações envolvendo o bullying. Verificou-se que 17,9\% dos brasileiros relataram que ninguém 
os defendeu quando sofreram bullying, contra apenas $6,9 \%$ dos portugueses que relataram a mesma situação (Tabela 3). A retroalimentação desse ciclo de violência se deve a incapacidade de a vítima denunciar a violência por sentir-se humilhada e com medo de sofrer novas violências ou violências mais graves, e também do espaço escolar, que é ineficiente na resolução do problema e muitas vezes assumindo a postura e o posicionamento de que o bullying é um fenômeno natural (Freire e Aires, 2012; Lopes Neto, 2005).

Tabela 3 - Informações das vítimas sobre contar a alguém da agressão sofrida e apoio recebido.

\begin{tabular}{l|c|c|c|c}
\hline \multirow{2}{*}{ Variáveis } & Total & Portugal & Brasil & \multirow{2}{*}{ p-valor* } \\
\cline { 2 - 4 } & $\mathrm{n}(\%)$ & $\mathrm{n}(\%)$ & $\mathrm{n}(\%)$ & \\
\hline $\begin{array}{l}\text { Você contou a alguém que alunos(as) } \\
\text { fizeram algum mal a você na escola? }\end{array}$ & & & & 0,012 \\
\hline Não & $78(18,5)$ & $27(13,7)$ & $51(22,7)$ & \\
\hline Sim & $344(81,5)$ & $170(86,3)$ & $174(77,3)$ & \\
\hline $\begin{array}{l}\text { Há alunos(as) que te } \\
\text { defenderam quando outros } \\
\text { tentaram fazer mal a você? }\end{array}$ & & & & $<0,001$ \\
\hline $\begin{array}{l}\text { Nenhum(a) aluno(a) fez } \\
\text { algo de mal para mim }\end{array}$ & $363(46,1)$ & $199(50,9)$ & $164(41,3)$ & \\
\hline Ninguém me defendeu & $98(12,4)$ & $27(6,9)$ & $71(17,9)$ & \\
\hline $\begin{array}{l}\text { Um(a) ou dois(duas) } \\
\text { alunos(as) me defenderam }\end{array}$ & $191(24,2)$ & $95(24,3)$ & $96(24,2)$ & \\
\hline Três ou mais alunos(as) & $136(17,3)$ & $70(17,9)$ & $66(16,6)$ & \\
\hline
\end{tabular}

n: número de participantes. * Qui-quadrado. Fonte: Banco de dados da pesquisa. Elaboração dos autores.

Sobre os espaços onde os participantes sofreram agressões, evidenciou-se que a maioria, em ambos os países, indicou o recreio como o lugar mais frequente na ocorrência de bullying (37,1\% em Portugal; 32,3\% no Brasil). Todavia, verificou-se que os participantes brasileiros relataram maior frequência de vitimização nas salas de aula que os portugueses $(22,7 \%$ e $12,3 \%$, respectivamente). De modo similar, os participantes brasileiros também relataram casos de bullying nos momentos de aula de educação física e na saída da escola (5,8\% e $7,1 \%$, respectivamente), enquanto os portugueses não citaram nenhuma ocorrência nesses espaços (Tabela 4). Para se compreender o fenômeno bullying, é necessário ter uma visão ecológica, compreendendo que o todo, o ecossistema escolar, está envolvido em sua origem e manutenção (Bronfenbrenner, 2011). Sendo assim, qualquer tipo de interferência nesse fenômeno deve levar em consideração diversas dimensões sociais e espaços de interação que a escola engloba (Freire e Aires, 2012). 
Tabela 4 - Lugares onde ocorrem as vitimizações.

\begin{tabular}{l|c|c|c|c}
\hline \multirow{2}{*}{$\begin{array}{l}\text { Em que lugar essas } \\
\text { situações acontecem? }\end{array}$} & Total & Portugal & Brasil & \multirow{2}{*}{ p-valor* } \\
\cline { 2 - 4 } & $\mathrm{n} \mathrm{( \% )}$ & $\mathrm{n} \mathrm{( \% )}$ & $\mathrm{n} \mathrm{( \% )}$ & \\
\hline Corredores e/ou escadas & $91(11,6)$ & $49(12,5)$ & $42(10,6)$ & 0,232 \\
\hline Recreio & $273(34,7)$ & $145(37,1)$ & $128(32,3)$ & 0,092 \\
\hline Sala de aula & $138(17,5)$ & $48(12,3)$ & $90(22,7)$ & $<0,001$ \\
\hline Refeitório e/ou cantina & $49(6,2)$ & $27(6,9)$ & $22(5,6)$ & 0,263 \\
\hline Casas de banho & $36(4,6)$ & $15(3,8)$ & $21(5,3)$ & 0,208 \\
\hline Educação física & $23(2,9)$ & $0(0,0)$ & $23(5,8)$ & $<0,001$ \\
\hline Saída da escola & $28(3,5)$ & $0(0,0)$ & $28(7,1)$ & $<0,001$ \\
\hline
\end{tabular}

n: número de participantes. *Qui-quadrado. Fonte: Banco de dados da pesquisa. Elaboração dos autores.

Com relação à frequência que os participantes agrediram alguém na escola, verificou-se que não houve associação dessa variável com o país de origem $(p=0,184)$. Foi identificado que em ambos os países a justificativa mais comum para tal agressão foi a necessidade de se defender (60,4\% em Portugal; 55,6\% no Brasil). Contudo, foi encontrada associação significativa entre a justificativa "porque me irritaram, provocaram ou gozaram" e o país de origem, sendo que a maior frequência foi encontrada entre os participantes brasileiros $(59,3 \%)$ (Tabela 5). O autor pode ser influenciado por condições adversas de sua família. Ele é popular e considera o comportamento agressivo uma qualidade. Utiliza o bullying para se sobressair socialmente, pois desconsidera a escola e a família como importantes (Freire e Aires, 2012).

Tabela 5 - Frequência em que agrediu alguém na escola e os motivos.

\begin{tabular}{l|c|c|c|c}
\hline \multirow{2}{*}{ Variáveis } & Total & Portugal & Brasil & \multirow{2}{*}{ p-valor* } \\
\cline { 2 - 4 } & $\mathrm{n}(\%)$ & $\mathrm{n}(\%)$ & $\mathrm{n}(\%)$ & \multirow{2}{*}{0,184} \\
\hline $\begin{array}{l}\text { Quantas vezes você fez mal } \\
\text { a outros(as) alunos(as) na } \\
\text { escola no último período? }\end{array}$ & & & & \\
\hline Nenhuma vez & $470(59,6)$ & $227(57,9)$ & $243(61,4)$ & \\
\hline Uma ou duas vezes & $249(31,6)$ & $122(31,1)$ & $127(32,1)$ & \\
\hline Três ou quatro vezes & $38(4,8)$ & $24(6,1)$ & $14(3,5)$ & \\
\hline Cinco ou mais vezes & $31(3,9)$ & $19(4,8)$ & $12(3,0)$ & \\
\hline Por que fizestes? & & & & \\
\hline$\quad$ Porque tive de me defender & $77(57,5)$ & $32(60,4)$ & $45(55,6)$ & 0,355 \\
\hline $\begin{array}{l}\text { Porque me irritaram, } \\
\text { provocaram ou gozaram }\end{array}$ & $69(51,5)$ & $21(39,6)$ & $48(59,3)$ & 0,020 \\
\hline
\end{tabular}

n: número de participantes. *Qui-quadrado. Fonte: Banco de dados da pesquisa. Elaboração dos autores. 
Também foi encontrada associação significativa entre a frequência relatada pelos participantes em ajudar a agredir um colega que não gostava e o país de origem $(\mathrm{p}=0,003)$. De acordo com os dados, os participantes portugueses relataram, em maior porcentagem, que não ajudariam a agredir um colega de quem não gostavam $(65,3 \% \mathrm{em}$ Portugal; 50,8\% no Brasil) (Tabela 6). O agressor pode possuir um grupo de seguidores para dividir a culpa da ação. Os seguidores geralmente fazem isso para se proteger de agressões, pois fazem parte de um grupo dominante (Freire e Aires, 2012).

Tabela 6 - Frequência sobre ajudar a agredir um colega.

\begin{tabular}{l|c|c|c|c}
\hline $\begin{array}{l}\text { Ajudavas a agredir } \\
\text { um(a) colega de quem } \\
\text { não gostavas? }\end{array}$ & Total & Portugal & Brasil & \multirow{2}{*}{ p-valor* } \\
\cline { 1 - 4 } & $\mathrm{n}(\%)$ & $\mathrm{n}(\%)$ & $\mathrm{n}(\%)$ & \\
\hline Não & $228(58,2)$ & $130(65,3)$ & $98(50,8)$ & \multirow{2}{*}{0} \\
\hline Acho que não & $52(13,3)$ & $30(15,1)$ & $22(11,4)$ & \multirow{2}{*}{0,003} \\
\hline Só se ele me irritar muito & $78(19,9)$ & $26(13,1)$ & $52(26,9)$ & \\
\hline Talvez & $22(5,6)$ & $8(4,0)$ & $14(7,3)$ & \\
\hline Sim & $12(3,1)$ & $5(2,5)$ & $7(3,6)$ & \\
\hline
\end{tabular}

n: número de participantes. *Qui-quadrado. Fonte: Banco de dados da pesquisa. Elaboração dos autores.

Por fim, foi verificada associação significativa entre os papéis de participação no bullying e a experiência de ficar isolado na escola $(\mathrm{p}=0,003)$. Observou-se que as vítimas-agressoras foram aquelas que mais relataram ficar isoladas três ou mais vezes nos últimos três meses (44\%), seguidas pelas vítimas $(28,6 \%)$ (Tabela 7). Esse dado é especialmente preocupante, pois as pesquisas demostram que os relacionamentos interpessoais positivos na escola têm relação direta com o bom desempenho do estudante; dessa forma, um relacionamento ruim pode resultar em desempenhos menos satisfatórios. Também a aceitação social possui relação com estado de saúde geral da criança e do adolescente, principalmente no que tange à saúde mental (Freire e Aires, 2012).

Tabela 7 - Associação entre papéis de participação no bullying e experiência de ficar isolado na escola.

\begin{tabular}{|c|c|c|c|c|c|}
\hline \multirow{2}{*}{$\begin{array}{l}\text { Quantas vezes você } \\
\text { ficou sozinho(a), } \\
\text { porque os outros } \\
\text { alunos(as) não } \\
\text { queriam a sua } \\
\text { companhia? }\end{array}$} & $\begin{array}{c}\text { Não } \\
\text { Participa }\end{array}$ & Vítima & Agressor & $\begin{array}{c}\text { Vítima- } \\
\text { Agressora }\end{array}$ & \multirow[b]{2}{*}{ p-valor* } \\
\hline & $\mathrm{n}(\%)$ & $\mathrm{n}(\%)$ & $\mathrm{n}(\%)$ & $\mathrm{n}(\%)$ & \\
\hline Nunca fiquei só & $176(69,3)$ & $45(45,9)$ & $10(50,0)$ & $7(28,0)$ & \multirow{3}{*}{0,003} \\
\hline $\begin{array}{l}\text { Uma ou duas vezes nos } \\
\text { últimos três meses }\end{array}$ & $61(24,0)$ & $25(25,5)$ & $9(45,0)$ & $7(28,0)$ & \\
\hline $\begin{array}{l}\text { Três ou mais vezes nos } \\
\text { últimos três meses }\end{array}$ & $17(6,7)$ & $28(28,6)$ & $1(5,0)$ & $11(44,0)$ & \\
\hline
\end{tabular}

n: número de participantes. *Qui-quadrado. Fonte: Banco de dados da pesquisa. Elaboração dos autores. 


\section{DISCUSSÃO}

Entre os principais achados deste estudo, verificou-se que entre as características gerais dos participantes os escolares brasileiros apresentaram maiores associações com menor escolaridade da mãe, maior frequência de reprovação, menor prática de atividades físicas, menos chances de denunciar o agressor e menos apoio dos colegas no momento da agressão. Em relação aos papéis de participação no bullying, os participantes brasileiros mais se autodeclararam vítimas, enquanto os participantes portugueses se disseram mais vítimas-agressoras. Contudo, verificou-se maior associação da frequência de vitimização leve e severa com os escolares brasileiros, e de vitimização média com os portugueses. Em ambos os países, o local mais citado onde ocorreram as agressões foi no recreio, mas verificou-se que os participantes do Brasil relataram também agressões na sala de aula, nos momentos de aula de educação física e na saída da escola, quando comparados aos participantes de Portugal. Por conseguinte, não foi encontrada associação entre a frequência que os participantes agrediram alguém na escola e o país de origem, mas os participantes brasileiros justificaram mais suas agressões em virtude de terem sido irritados, provocados ou gozados, bem como também foram aqueles que mais relataram que ajudariam a agredir um colega de quem não gostassem. Por fim, quando analisados os papéis de participação no bullying, de todos os participantes, encontrou-se associação entre ficar isolado e ser vítima-agressora.

Compreende-se que o bullying não é um problema apartado ou específico de uma única cultura, mas sim um tipo de violência presente em escolas do mundo todo (Cook et al., 2010). Contudo, com base nos resultados apresentados, pode-se afirmar que, aparentemente, os participantes brasileiros deste estudo apresentaram maiores fatores de risco para o envolvimento nesse fenômeno, especialmente no que tange às chances de vitimização pelos pares. Chegou-se a tal conclusão em virtude de alguns resultados coincidirem com determinados fatores indicados na literatura que podem favorecer o envolvimento no bullying escolar, entre os quais se podem destacar: baixa escolaridade dos pais (Analitis et al., 2009; Perren, Stadelmann e Klitzing, 2009); desempenho escolar deficiente e altos índices de reprovação (Álvarez-García, García e Núñez, 2015; Holt, Finkelhor e Kantor, 2007; Matos et al., 2009), pior desempenho motor e pouca participação em atividades físicas. Com relação à participação no bullying, verificou-se que a porcentagem de envolvimento foi aproximadamente de $25 \%$ dos participantes em ambos os países, número este que está de acordo com a literatura, a qual sugere que entre $10 \%$ e $35 \%$ de crianças e adolescentes em idade escolar estejam envolvidos nesse fenômeno (Solberg e Olweus, 2003). Em relação aos papéis, verificou-se maior frequência de escolares brasileiros enquanto vítimas e de portugueses enquanto vítimas-agressoras. Alguns estudos transculturais também encontraram diferenças entre os países no que tange aos papéis de participação no bullying. No estudo de Wolke et al. (2001), encontraram-se diferenças de vitimização entre crianças alemãs e inglesas. Da mesma forma, no estudo de Smith-Khuri et al. (2004) os escolares da Irlanda, Israel, Portugal, Suécia e Estados Unidos também apresentaram diferenças entre os países, especificamente em comportamentos violentos relacionados ao bullying, sugerindo que esse fenômeno pode ser mais suscetível 
às influências culturais e ambientais do que outros comportamentos relacionados a atos violentos. Para explicar tais diversidades encontradas nesses estudos transculturais, alguns autores apontam as características culturais, as diferenças das escolas e rendas desiguais entre os países como possíveis fatores que podem ter influência sobre fenômenos como o bullying (Harel-Fisch et al.,2011; Smith-Khuri et al.,2004; Wolke et al., 2001).

Os participantes brasileiros também foram os que menos contaram para alguém sobre a agressão sofrida e que menos receberam ajuda no momento da vitimização. Provavelmente, esse fato está relacionado à maior porcentagem de vítimas nesse país. Sabe-se que as vítimas, geralmente, constituem o grupo com poucos amigos, são passivos, retraídos, pouco sociáveis, inseguros, sofrem com a vergonha, medo, depressão, ansiedade, e são desesperançados quanto à possibilidade de adequação ao grupo (Carvalhosa, Lima e Matos, 2001; Smith e Sharp, 1994). São aqueles que têm piores relações com os pares e sua baixa autoestima é agravada por críticas dos adultos à sua vida ou comportamento, dificultando a possibilidade de ajuda (Lopes Neto, 2005). Ademais, o envolvimento no bullying está fortemente associado ao aumento da internalização de problemas (Molina et al., 2014), e crianças e adolescentes rejeitados tendem a receber menos ajuda e mais críticas dos professores, razão pela qual muitas vezes eles deixam de procurá-los, pois acreditam que isso não iria resolver, mas sim agravar a situação de vitimização (Estéveza et al., 2014; Newman e Murray, 2005). Assim, também é necessário maior preparo por parte dos professores, a fim de evitar que reforcem os sentimentos de inferioridade das vítimas, bem como a incredulidade com relação ao fim do sofrimento por elas vivenciado. De mesmo modo, também é importante maior conscientização dos colegas não envolvidos no bullying, pois é sabido o quão fundamental é para as vítimas ter alguém as defenda. A presença de um único amigo para defender já faz com que a vítima reduza a ansiedade e a depressão, melhore a autoestima, além de diminuir a frequência da ocorrência de bullying e seus efeitos em curto e longo prazo (Molina, Valero e Canales, 2011; Sainio et al., 2010).

As características da amostra brasileira que indicam maior risco para o envolvimento em situações de bullying podem ser analisadas considerando-se o funcionamento cultural do país. No Brasil, um dos fatores determinantes para o estabelecimento do status social é o nível socioeconômico (Levandoski e Cardoso, 2013), o que significa que pessoas financeiramente menos favorecidas são consideras inferiores ou menos capazes. Então pessoas nessas circunstâncias aceitam sua condição de inferioridade, o que acaba por dificultar a possibilidade de reação aos processos de violência dos quais são atores. A baixa escolaridade dos pais e o fraco desempenho escolar do filho são parte desses fenômenos, uma vez que pais que acreditam não possuírem capacidade suficiente para mudar seu status social transmitem essa ideologia para seus filhos, que já entram no sistema escolar acreditando em sua incapacidade (Fonseca, Medeiros e Cavalcante, 2016). Essa reprodução ideológica faz com que aceitem com facilidade o não aprender e os processos de violência, considerando que a culpa dessa condição está relacionada às suas características pessoais. O que também justificaria a maior prevalência de brasileiros vítimas, se comparados com a amostra portuguesa (Guareschi, 2000). 
Os diversos tipos de bullying vêm ocorrendo dentro de todo o ambiente escolar e de suas imediações, principalmente nos espaços e tempos livres dos alunos (Lourenço et al., 2009). Neste estudo, verificou-se que a maior ocorrência de bullying se deu no recreio, tanto em Portugal $(37,1 \%)$ quanto no Brasil $(32,3 \%)$. Esses resultados são semelhantes ao encontrado por Lourenço et al. (2009), no qual as agressões no recreio também foram mais prevalentes (31,5\%). Contudo, foi identificado que outros locais tiveram mais situações de agressões entre os participantes do Brasil, o que possivelmente indica uma menor supervisão desses espaços nas escolas brasileiras, pois sabe-se que locais inseguros e pouco supervisionados facilitam a ocorrência de bullying (Lopes Neto e Saavedra, 2003). Assim, as comunidades escolares devem se preocupar em desenvolver ambientes físicos e sociais seguros e implementar propostas que ajudem os alunos a aprender e adotar escolhas saudáveis (Levasseur, Kelvin e Grosskopf, 2013; Roman e Taylor, 2013).

Uma das possíveis explicações para a menor supervisão dos espaços das escolas brasileiras pode decorrer da invisibilidade social do fenômeno bullying no Brasil. Embora já existam vários estudos que dão visibilidade a esse tema, a apropriação dos conceitos que envolvem o fenômeno na comunidade escolar ainda é ínfima. Sendo assim, esses processos violentos nas escolas acabam não percebidos como algo a ser combatido e como uma causa de sofrimento psíquico. A invisibilidade da ocorrência do bullying nas escolas pode ser percebida pela negação ou ausência de tomada de posição diante do problema por professores e, consequentemente, também pelos demais membros da comunidade escolar (Da Silva et al., 2015; Trevisol e Campos, 2016) e pela minimização dos atos violentos no uso de explicações que os justificam como algo natural ou decorrente da faixa etária dos alunos, ou como característica de relações sociais e interpessoais. Assim, a importância e a ocorrência das ações de bullying, os atores envolvidos, as consequências de suas ações na saúde tornam-se também não visíveis (Gonçalves, 2007; Guareschi, 2000; Vieira, 2006).

Em contrapartida, não foi encontrada associação entre a frequência em que os participantes agrediram os colegas e o país de origem. Todavia, houve maior frequência de agressões em virtude de terem sido irritados, provocados ou gozados, por parte dos participantes brasileiros. Da mesma forma, também foram os brasileiros quem mais ajudariam a agredir um colega de quem não gostassem. Embora não se tenha encontrado diferenças na frequência de agressões entre os países, essas características dos escolares brasileiros podem constituir fatores de risco para futuro envolvimento no bullying. Torna-se necessário determinar quais fatores de risco podem predizer o envolvimento nesse fenômeno no papel de agressor, pois só assim se poderá identificar precocemente as crianças que ocupam lugar de risco, impedindo que se tornem agressores na adolescência e idade adulta, bem como poderão auxiliar na elaboração de estratégias de prevenção e intervenção desse tipo de violência na escola (Álvarez-García, García e Núñez, 2015).

Em seu estudo, Levandoski e Cardoso (2013) constataram que os agressores apresentavam maior status social em sala de aula e eram avaliados como mais atraentes pelo o sexo oposto. Já as vítimas e agressores/vítimas tinham menor status social e eram considerados menos atraentes pelo sexo oposto. Sendo assim, estabelecidas essas hierarquias sociais, os alunos sem envolvimento com o bullying tinham medo de se 
relacionar com a vítima e atrair seu papel para si. Considerando esse aspecto, pode-se analisar que ajudar a agredir um colega que é vítima ou responder à sua frustração com agressividade são comportamentos que aproximam o indivíduo do status social mais elevado na hierarquia social da escola. Intervenções que foquem no desenvolvimento de habilidades sociais e no fortalecimento da diversidade humana no contexto escolar poderiam impactar o bullying por reestruturar a hierarquia social dos estudantes e, consequentemente, criar o desejo de identificação com pessoas com comportamentos que não causam danos aos demais (Fonseca, Medeiros e Cavalcante, 2016).

Estudos apontam que o isolamento, a rejecção ou a falta de amigos podem estar associados à vitimização no bullying, pois crianças rejeitadas são mais frequentemente intimidadas, enquanto crianças com mais amigos são menos propensas à vitimização (Babarro, 2014; Salmivalli, 2010). Por esse motivo, este estudo buscou analisar também possíveis associações entre os papéis de participação no bullying e a experiência de ficar isolado na escola. Os resultados encontrados indicaram maior associação entre ficar isolado e o papel de vítima-agressora. Embora muitos estudos negligenciem esses participantes, sabe-se que vítimas-agressoras são menos competentes socialmente e apresentam características que dificultam o estabelecimento de boas amizades, como apoio emocional e equilibrio nas interações recíprocas, fazendo com que elas também sejam menos procuradas e aceitas pelos colegas (Kochel et al.,2015). Sabendo-se que esse é o grupo no qual se encontram maiores fatores de risco, os quais tem um efeito não apenas aditivo, mas multiplicativo (Spence e Matos, 2000), intervenções focadas em vítimas-agressoras devem ser priorizadas (Kochel et al.,2015). Esses dados apontam que o contexto escolar, em seu funcionamento, acaba por reproduzir o sistema social individualista e competitivo vigente na cultura atual. Porém, esses processos acabam não contribuindo para uma importante função no desenvolvimento psíquico da criança e do adolescente, que é responsabilidade da escola: o desenvolvimento de vínculos afetivos entre os que ali convivem, além de propiciar um espaço que beneficie a interação entre pessoas externas ao núcleo de socialização primária, contribuindo para a construção de suportes sociais que possam ser mantidos por toda a vida.

Por fim, traz-se como limitações deste estudo:

- a realização de uma pesquisa não probabilística não permite que os resultados encontrados possam ser estendidos de maneira geral a toda a população de ambos os países;

- o delineamento transversal do estudo impede a realização de inferências sobre a direção das associações identificadas, não permitindo o estabelecimento de causalidades;

- o perfil de espectador não pode ser analisado neste estudo em virtude da escolha do instrumento para comparações com outros resultados; contudo, ressalta-se a importância dos espectadores para as práticas de intervenção a serem realizadas na escola;

- a pesquisa foi realizada em duas realidades (Portugal e Brasil) que, apesar de possuírem características diferentes, são de um mesmo tronco “judaico-cristão" e origem, aspecto este que pode interferir nas análises e resultados de pesquisas de cunho intercultural em que essas semelhanças não sejam observadas. 
Salienta-se a importância de que pesquisas futuras possam adotar delineamento longitudinal em estudos transculturais, possibilitando o acompanhamento do fenômeno ao longo do tempo, suprindo a limitação do presente estudo, além de fornecer subsídios para prevenção e combate à violência escolar em diferentes países. Todavia, mesmo diante das limitações citadas, os resultados acentuam a relevância relacionada ao tema, apresentando indícios de que o fenômeno bullying caracteriza-se como um grave problema de saúde pública no âmbito escolar. Esse problema acentua-se em virtude da sua elevada prevalência e dos prejuízos para o desenvolvimento saudável de crianças e adolescentes, seja tanto nas esferas afetivas quanto social, e das relações entre os pares (De Oliveira et al., 2015). Além disso, com os resultados obtidos foi possível estabelecer um quadro mais completo em relação ao bullying escolar em ambos os países. Verificou-se que identificar o bullying escolar em suas diferentes formas de manifestação e o modo como afetam a vida das crianças e adolescentes são aspectos fundamentais para oferecer o cuidado e o suporte necessário.

\section{CONSIDERAÇÕES FINAIS}

O presente estudo apresenta informações relevantes no que tange ao panorama geral sobre o bullying em participantes de Portugal e do Brasil. Por meio dos resultados apresentados foi possível verificar associações entre alguns aspectos desse fenômeno e o país de origem. De modo geral, os participantes brasileiros apresentaram maiores indícios de vulnerabilidade para o envolvimento nesse comportamento violento. Ademais, o estudo também salientou uma característica de risco presente em crianças e adolescentes vítimas-agressoras, que é o isolamento social na escola. Sabendo-se que aqueles que assumem tal papel são os que apresentam maiores fatores de risco, identificá-los por meio da característica do isolamento social pode ser mais uma ferramenta utilizada por professores e funcionários para auxiliar no combate a esse tipo de comportamento violento. Essa rejeição pelos pares, aliada a outros fatores típicos desse perfil, reforça a necessidade de mais estudos voltados para compreendê-los, a fim de fornecer maiores evidências para a elaboração de programas de prevenção e intervenção mais eficazes.

\section{REFERÊNCIAS}

ÁLVAREZ-GARCÍA, D.; GARCÍA,T.; NÚÑEZ,J.C. Predictors of school bullying perpetration in adolescence: a systematic review. Aggression and Violent Behavior, Amsterdam, v. 23, p. 126-136, 2015. http://dx.doi.org/10.1016/j.avb.2015.05.007

ANALITIS, F. et al. Being bullied: associated factors in children and adolescents 8-18 years old in 11 European countries. Pediatrics, Itasca, v. 123, p. 569-577, 2009. http://dx.doi.org/10.1542/peds.2008-0323

BABARRO, J. M. Assessment and detection of peer-bullying through analysis of the group context. Psicothema, Spain, v. 26, n. 3, p. 357-363, Aug. 2014. http://dx.doi.org/10.1037/t61647-000 
BRONFENBRENNER, U. Bioecologia do desenvolvimento humano: tornando os seres humanos mais humanos. Porto Alegre: Artmed Editora, 2011.

CARVALHOSA, S. D.; LIMA, L.; MATOS, M. D. Bullying: a provocação/vitimação entre pares no contexto escolar português. Análise Psicológica, Lisboa, v. 4, n. 19, p. 523-537, 2001.

CENTERS FOR DISEASE CONTROL AND PREVENTION. Bullying surveillance among schoolaged children: Uniform definitions and recommended data elements. Washington, DC: Centers for Disease Control and Prevention (CDC), 2014. COOK, C. et al. Predictors of childhood bullying and victimization: a meta-analytic review. School Psychology Quarterly, Washington, DC, v. 25, n. 2, p. 65-83, 2010. http://dx.doi.org/10.1037/a0020149

CRAIG, W. et al. A cross-national profile of bullying and victimization among adolescents in 40 countries. International Journal of Public Health, [S.l.], v. 54, suppl. 2, p. 216-24, 2009. Disponível em: http://www.ncbi.nlm.nih.gov/pubmed/19623475. Acesso em: 23 fev. 2019. http://dx.doi.org/10.1007/s00038-009-5413-9

DA SILVA,J. L.et al.Estudo exploratório sobre as concepções e estratégias de intervenção de professores em face do bullying escolar. Psicologia: teoria e prática, São Paulo, v. 17, n. 3, 2015. http://dx.doi.org/10.15348/1980-6906/psicologia.v17n3p189-199

DE OLIVEIRA, W. A. et al. The causes of bullying: results from the National Survey of School Health (PeNSE). Revista Latino-Americana de Enfermagem, Ribeirão Preto, v. 23, n. 2, p. 275-82, 2015 Mar./Apr. 2015. Disponível em: http://www.ncbi.nlm.nih.gov/pubmed/26039298. Acesso em: 23 fev. 2019. http://dx.doi.org/10.1590/0104-1169.0022.2552

ESTÉVEZA, E. et al. Psychosocial adjustment in aggressive popular and aggressive rejected adolescents at school. Psychosocial Intervention, Madrid,v.23, p. 57-67, 2014. http://dx.doi.org/10.5093/in2014a6

FOnSECA, T. D. S.; MEDEIROS, C. M. L. D.; CAVAlCANTE, A. C. S. Habilidades sociais de amizade e civilidade no contexto escolar. Estudos Interdisciplinares em Psicologia, Londrina, v. 7, n. 2, p. 147-156, 2016. http://dx.doi.org/10.5433/2236-6407.2016v7n2p147

FREIRE, A.; AIRES, J. A contribuição da psicologia escolar na prevenção e no enfrentamento do bullying. Psicologia Escolar e Educacional, Maringá, v. 16, n. 1, p. 55-60, 2012. http://dx.doi.org/10.1590/S1413-85572012000100006

GAFFNEY, H.; TTOFI, M. M.; FARRINGTON, D. P. Evaluating the effectiveness of school-bullying prevention programs: an updated metaanalytical review. Aggress Violent Behav, Amsterdam, 2018. http://dx.doi.org/10.1016/j.avb.2018.07.001

GONÇALVES, J. L. Invisibilidade e reconhecimento: a construção da literacia moral em pedagogia social. Cadernos de Pedagogia Social 1, Porto, p. 83-103, 2007.

GUARESCHI, P. A. Representações sociais e ideologia (Social Representations and Ideology). Revista de Ciências Humanas, Florianópolis, n. 3, p. 33-46, 2000. https://doi.org/10.5007/\%25x 
HAREL-FISCH, Y. et al. Negative school perceptions and involvement in school bullying: a universal relationship across 40 countries. Journal of Adolescence, Amsterdam, v. 34, n. 4, p. 639-652, Aug 2011. Disponível em: http://www.ncbi.nlm.nih.gov/pubmed/21168202. Acesso em: 23 fev. 2019. http://dx.doi.org/10.1016/j.adolescence.2010.09.008

HOLT,M. K.; FINKELHOR,D.; KANTOR, G. K. Multiple victimization experiences of urban elementary school students: associations with psychosocial functioning and academic performance. Child Abuse \& Neglect, Amsterdam, v. 31, n. 5, p. 503-15, May 2007. Disponível em: http://www.ncbi.nlm.nih.gov/pubmed/17537507. Acesso em: 23 fev. 2019. http://dx.doi.org/10.1016/j.chiabu.2006.12.006

KOCHEL, K. P. et al. Bully/victim profiles' differential risk for worsening peer acceptance: the role of friendship. Journal of Applied Developmental Psychology, Amsterdam, v. 41, p. 38-45, 2015 Nov./Dec. 2015. Disponível em: http://www.ncbi.nlm.nih.gov/pubmed/26309346. Acesso em: 23 fev. 2019. http://dx.doi.org/10.1016/j.appdev.2015.05.002

LEVANDOSKI, G.; CARDOSO, F. Imagem corporal e status social de estudantes brasileiros envolvidos em bullying. Revista Latinoamericana de Psicología, Bogotá, v. 45, n. 1, p. 135-45, 2013.

LEVASSEUR, M. T.; KELVIN, E. A.; GROSSKOPF, N. A. Intersecting identities and the association between bullying and suicide attempt among New York city youths: results from the 2009 New York city youth risk behavior survey. American Public Health Association, Washington, DC, v. 103, n. 6, p. 1082-9, Jun 2013. Disponível em: http://www.ncbi.nlm.nih.gov/pubmed/23597376. Acesso em: 23 fev. 2019. http://dx.doi.org/10.2105/AJPH.2012.300994

LOPES NETO, A. Bullying: comportamento agressivo entre estudantes. Jornal de Pediatria, Rio de Janeiro, v. 81, n. 5, p. 164-72, 2005. http://dx.doi.org/10.1590/S0021-75572005000700006. LOPES NETO,A.; SAAVEDRA,L.Diga não ao bullying. Rio de Janeiro: ABRAPIA,2003. LOURENÇO, L. et al. A gestão educacional e o bullying: um estudo em escolas portuguesas. Interacções, Lisboa, n. 13, p. 208-28 2009.

MATOS, M. et al. Violência, bullying e delinquência. 1. ed. Lisboa: Gráfica Manuel Barbosa \& Filhos, 2009.

MODECKI, K. L. et al. Bullying prevalence across contexts: a meta-analysis measuring cyber and traditional bullying. Journal of Adolescent Health, New York, n. 55, p. 602611, 2014. http://dx.doi.org/10.1016/j.jadohealth.2014.06.007

MOLCHO, M. et al. Cross-national time trends in bullying behaviour 1994-2006: findings from Europe and North America. International Journal of Public Health, Switzerland, v. 54, n. 2, p. 225-234, 2009. http://dx.doi.org/10.1007/s00038-009-5414-8 MOLINA, B. L.; VALERO, R. P.; CANALES, I. S. Violencia entre iguales en Educación Primaria:el papel de los compañeros y su relación con el estatus sociométrico Psicothema, Spain, v. 23, n. 2, p. 245-51, 2011.

MOLINA, B. L. et al. Adaptation of the Participant Role Scale (PRS) in a Spanish youth sample: measurement invariance across gender and relationship with sociometric 
status. Journal of Interpersonal Violence, Thousand Oaks, v. 29, n. 16, p. 2.9042.930, 2014. https://doi.org/10.1177/0886260514527822

NEWMAN, R. S.; MURRAY, B. J. How students and teachers view the seriousness of peer harassment: when is it appropriate to seek help? Journal of Educational Psychology, Washington, DC, v. 97, n. 3, p. 347-365, 2005. http://dx.doi.org/10.1037/0022-0663.97.3.347

OBRDALJ, E. C. et al. Trauma symptoms in pupils involved in school bullying: a cross sectional study conducted in Mostar, Bosnia and Herzegovina. Collegium antropologicum, Croatia, v. 37, n. 1, p. 11-16, Mar. 2013. Disponível em: http://www.ncbi.nlm.nih.gov/pubmed/23697244. Acesso em: 23 fev 2019.

OLWEUS, D. The revised Olweus Bully/Victim Questionnaire. Bergen: University of Bergen; Research Center for Health Promotion, 1996.

OLWEUS, D. Bully/victim problems among schoolchildren: basic facts and effects of a school based intervention program. In: PEPLER, D. J.; RUBIN, K. H. (eds.). The development and treatment of childhood aggression, Hillsdale, NJ, US: Lawrence Erlbaum Associates, 1991. p. 411-48.

PERREN, S.; STADELMANN, S.; KLITZING, K. Child and family characteristics as risk factors for peer victimization in kindergarten. Schweizerische Zeitschrift für Bildungswissenschaften, Zurich, v. 31, n. 1, p. 13-32, 2009.

ROMAN, C. G.; TAYLOR, C. J. A multilevel assessment of school climate, bullying victimization, and physical activity.Journal School Health, Bloomington, v. 83, n. 6, p. 400-407,Jun. 2013. Disponível em: http://www.ncbi.nlm.nih.gov/pubmed/23586884. Acesso em: 23 fev. 2019. http://dx.doi.org/10.1111/josh.12043

SAINIO, M. et al. Victims and their defenders: a dyadic approach. International Journal of Behavioral Development, Thousand Oaks, Oct 15, 2010. https://doi.org/10.1177/0165025410378068

SALMIVALLI, C. Bullying and the peer group: a review. Aggression and Violent Behavior, Amsterdam, v. 15, n. 2, p. 112-120, 2010. http://dx.doi.org/10.1016/j.avb.2009.08.007

SALMIVALLI, C.; POSKIPARTA, E. KiVa Antibullying Program: overview of evaluation studies based on a randomized controlled trial and national rollout in Finland. International Journal of Conflict and Violence, Germany, v. 6, n. 2, p. 294302, 2011.

SMITH, P.; SHARP, S. School bullying: insights and perspectives. London: Routledge, 1994.

SMITH-KHURI, E. et al. A cross-national study of violence-related behaviors in adolescents. Archives of Pediatrics and Adolescent Medicine, United States, v. 158, n. 6, p. 539-44,Jun. 2004. Disponível em: http://www.ncbi.nlm.nih.gov/pubmed/15184216. Acesso em: 23 fev. 2019. http://dx.doi.org/10.1001/archpedi.158.6.539

SOLBERG, M. E.; OLWEUS, D. Prevalence estimation of school bullying with the Olweus Bully/Victim Questionnaire. Aggressive Behavior, United Kingdom, v. 29, n. 3, p. 239-268, 2003. https://doi.org/10.1002/ab.10047 
SPENCE, S.; MATOS, M. Intervenções preventivas com crianças e adolescentes. In: MATOS, M.; SIMÕES, C. et al. (ed.). Desenvolvimento de competências de vida na prevenção do desajustamento social. Lisboa: IRS/MJ, 2000.

TREVISOL, M. T. C.; CAMPOS, C. A. Bullying: verificando a compreensão dos professores sobre o fenômeno no ambiente escolar. Psicologia Escolar e Educacional, São Paulo, v. 20, n. 2, p. 275-284, 2016. http://dx.doi.org/10.1590/2175-353920150202964

TSANG, S. K.; HUI, E. K.; LAW, B. C. Bystander position taking in school bullying: the role of positive identity, self-efficacy, and self-determination. The Scientific World Journal, London, v. 11, p. 2.278-2.286, 2011. Disponível em: http://www.ncbi.nlm.nih.gov/pubmed/22194663. Acesso em: 23 fev 2019. http://dx.doi.org/10.1100/2011/531474

VIEIRA, M. M. Em torno da família e da escola: pertinência científica, invisibilidade social. Interacções, Lisboa, v. 2, n. 2, p. 291-305, 2006.

WOLKE, D. et al. Bullying and victimization of primary school children in England and Germany: prevalence and school factors. British Journal of Psychology, United Kingdom, v. 92, n. Pt 4, p. 673-696, Nov. 2001. Disponível em: http://www.ncbi.nlm.nih.gov/pubmed/11762868. Acesso em: 23 fev 2019. https://doi.org/10.1348/000712601162419

ZEQUINÃO, M. A. et al. School bullying: a multifaceted phenomenon. Educação e Pesquisa, São Paulo, v. 42, n. 1, p. 181-198, 2016. http://dx.doi.org/10.1590/S15179702201603138354

ZYCH, I.; FARRINGTON, D. P.; LLORENT, V. J.; TTOFI, M. Protecting children against bullying and its consequences. Switzerland: Springer Briefs in Behavioral Criminology, 2017. http://dx.doi.org/10.1007/978-3-319-53028-4

\section{SOBRE OS AUTORES}

Marcela Almeida Zequinão é doutora em estudos da criança pela Universidade do Minho (Portugal). Professora da Universidade do Estado de Santa Catarina (UDESC). E-mail: marcelazequinao@gmail.com

Pâmella de Medeiros é doutoranda em ciências do movimento humano pela Universidade do Estado de Santa Catarina (UDESC). Professora da mesma instituição.

E-mail: pamellademedeiros@hotmail.com

Fábio Augusto Lise é mestre em saúde coletiva pela Universidade do Vale do Rio dos Sinos (UNISINOS). Professor da Universidade do Oeste de Santa Catarina (UNOESC).

E-mail: fabio.lise@unoesc.edu.br 
Maria Teresa Ceron Trevisol é doutora em psicologia escolar e do desenvolvimento humano pela Universidade de São Paulo (USP). Professora da Universidade do Oeste de Santa Catarina (UNOESC).

E-mail: mariateresa.trevisol@unoesc.edu.br

Maria Beatriz Ferreira Leite Oliveira Pereira é doutora em estudos da criança pela Universidade do Minho (Portugal). Professora da mesma instituição.

E-mail: beatriz@ie.uminho.pt

Recebido em 21 de maio de 2018 Aprovado em 11 de setembro de 2018 